\title{
CATECHIN OXIDATION PRODUCTS: MECHANISTIC ASPECTS AND KINETICS
}

\author{
ANCA - RUXANDRA LEONTIEŞ ${ }^{a *}$, ADINA RĂDUCAN ${ }^{b}$, IOANA \\ CATALINA GÎFU', DAN FLORIN ANGHEL ${ }^{a}$
}

\begin{abstract}
The oxidation of catechin in the presence of atmospheric oxygen in a methanol/buffer model solution was studied by investigating generated products using LC/MS and spectrophotometry. It was proved that catechin was slowly converted to dimeric species with similar structures and properties. The catechin autoxidation was investigated spectrophotometrically at different $\mathrm{pH}$ ranging between 5 and 10 . Using the initial linear dependence of the kinetic curves [Dimers] $=f(t)$ the initial reaction rates were estimated for the autoxidation of catechin in methanol/buffered solutions. It was found that the dimer formation is favored at $\mathrm{pH} 8$.
\end{abstract}

Keywords: catechin, autoxidation, dehydrocatechin

\section{INTRODUCTION}

Polyphenols are widely distributed in plants and in plant-derived products, mostly in wines and teas, where they contribute to food organoleptic properties and oxidative stability [1]. Polyphenols have several health benefits, acting as antioxidant, anticarcinogen, antimicrobial, anti-inflammatory and UV protection agents [2].

One of the most common natural polyphenols from the class of flavanols is catechin (Scheme 1). As polyphenol, catechin is susceptible to enzymatic and non-enzymatic oxidation. The oxidation products are the oxidation dimers. The dimers are linked either by $\mathrm{C} 6 \rightarrow \mathrm{C} 8$ or C $6 \rightarrow$ C6 interflavan linkages

\footnotetext{
a Ilie Murgulescu Institute of Physical Chemistry, Spl. Independentei 202, Bucharest, 060021, Romania

b University of Bucharest, Faculty of Chemistry, Bulevardul Regina Elisabeta 4-12, Bucharest, 030018, Romania

*Corresponding author: ancaleonties@gmail.com
} 
and are classified as B-type dehydrodicatechins, resulting from the repeated condensation reactions between the A-ring of the lower unit and the B-ring of the upper unit through a mechanism of so-called 'head to tail' polymerization [3] or they contain additional $\mathrm{C}-\mathrm{O}-\mathrm{C}$ ether-type interflavan linkages and are classified as A-type dehydrodicatechins [4].

During the past decades many studies focused on the protective effects on flavanols against lipid peroxidation and low-density lipoproteins, oxidation as well as on their antiproliferative and anticarcinogenic actions $[5,6]$. On the other hand, polyphenols are susceptible to autoxidation. In this process are generated large amounts of free radicals and active oxygen species, such as hydrogen peroxide, which are able to induce DNA damage and diseases $[7,8]$. Therefore, much discussion and contradiction exist regarding the structure and activity of oxidation products resulting from the autoxidation process.

In the present paper, we describe the autoxidation of catechin in aqueous/methanol model solution under $\mathrm{pH}$ ranging between 5 and 10 . On the basis of the LC-MS and HPLC we identified a series of B-type and A-type dimeric autoxidation products and proposed their structures and dissociation mechanisms. Furthermore, using a simple spectrophotometrical method we analyzed the kinetics of catechin autoxidation.

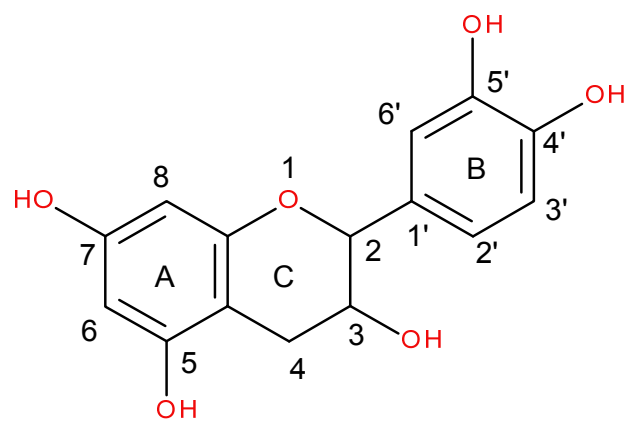

Scheme 1. Catechin structure

\section{RESULTS AND DISCUSSION}

The autoxidation of catechin was carried out in aqueous/ methanol solution under mildly basic conditions ( $\mathrm{pH} 8.0)$. By using a LC-MS system the autoxidation products were identified (Figure 1). 


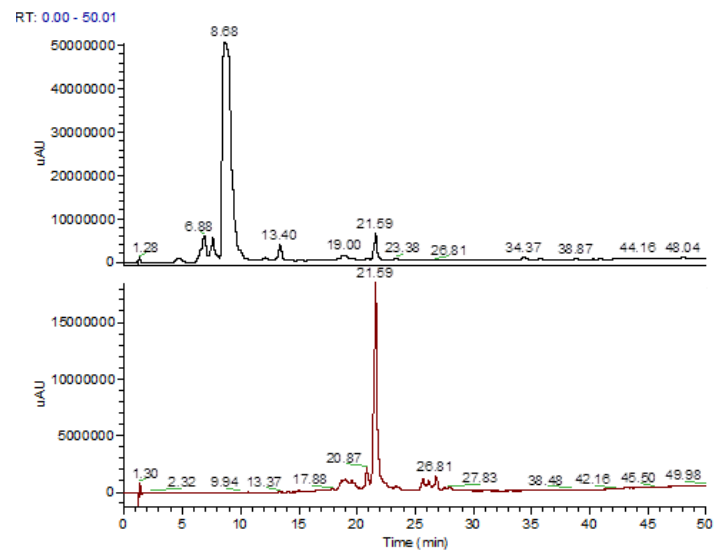

Figure 1. LC-MS cromatogram for the catechin autooxidation products

The peaks of the compounds eluted at retention times of 6.88 and 7.60 minutes were assigned to a dimer product resulted from autoxidation. These compounds have the same UV-Vis absorption spectrum with double maxima at 240 and $280 \mathrm{~nm}$ and similar ion fragments (Figure 2. A and B).
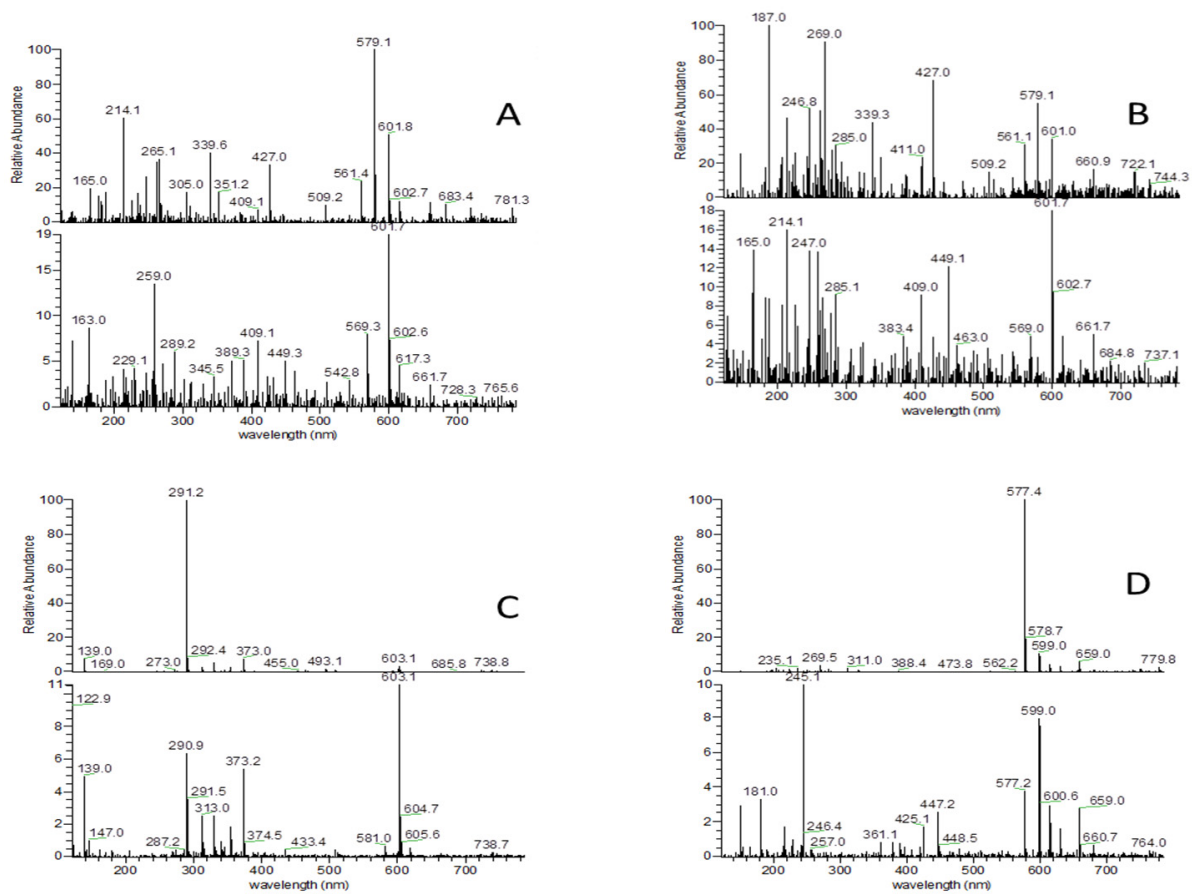

Figure 2. Mass spectra of identified catechin autoxidation products (retention time 6.88 minute - A, retention time 7.60 minutes - $B$, retention time 8.68 - C, retention time 21.59 D) 
At retention time 8.68 minutes the peak of the eluted compound was assigned to the remaining non-reactive catechin. Figure 2.B shows the majority ion with mass $\mathrm{m} / \mathrm{z} 291.2$ typical for catechin. The compound eluted at retention time 13.40 minutes corresponds to catechin which lost a hydrogen atom, this fact being confirmed by the presence in the mass spectrum of the fragment ion with $\mathrm{m} / \mathrm{z} 290$ (Figure 2.C). The compound with the peak at the retention time of 21.59 minutes (Figure 2.D) was established as another type of dimer product due to the presence in the mass spectrum of the fragment $m / z 577.4$. It has also been observed that the products with peaks at retention times 18.64 , 19.57 and 26.81 minutes have the same UV-Vis spectrum. Three maxima at 240, 256 and $376 \mathrm{~nm}$ in absorption spectrum were identified and similar ionic fragments to the compound found at retention time of 21.59 minutes. These compounds were supposed to be fragments of dimers resulting from MS fragmentation. During the autoxidation process, the B-type dehydrocatechin results from repeated condensation reactions between the A-ring of the lower unit and the B-ring of the upper unit in a 'head to tail' polymerization. At 6.88 and 7.60 minutes the elution peak was attributed to this type of dimer. The mechanistic formation of $\mathrm{m} / \mathrm{z} 579=\mathrm{M}+1$ and $\mathrm{m} / \mathrm{z}$ 601= $\mathrm{M}+23\left(\mathrm{M}_{\mathrm{Na}+}\right)$ sustains this idea. The supposed fragmentation path of B-type dehydrocatechin is presented in Scheme 2.
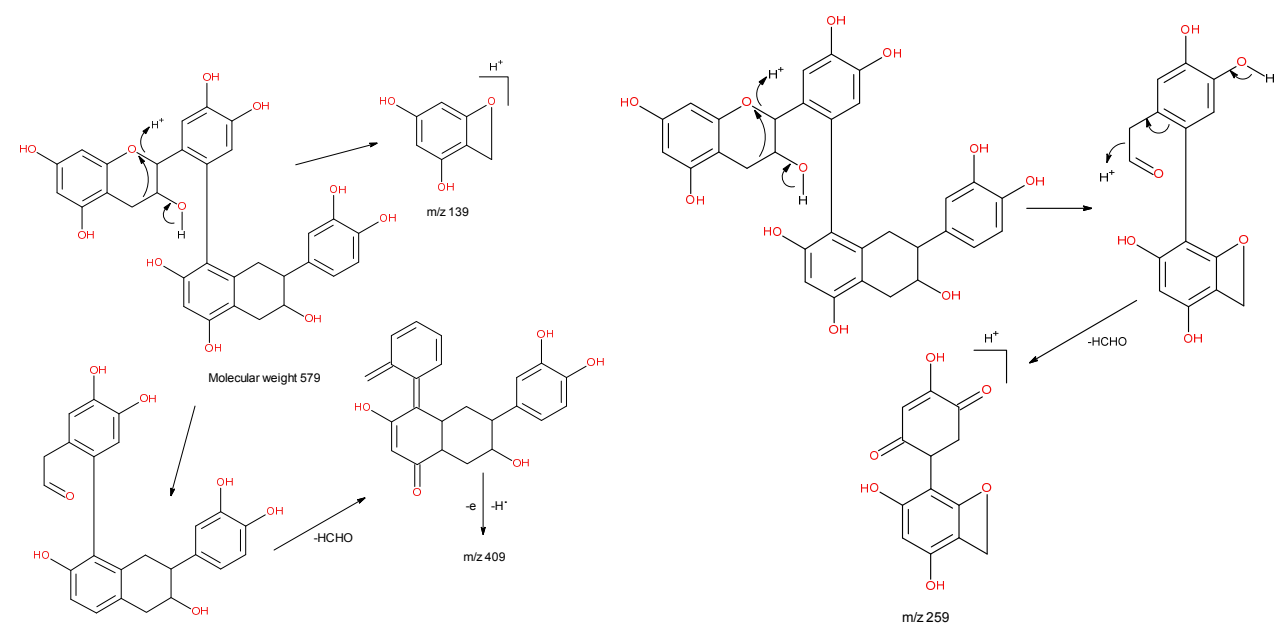

Scheme 2. Fragmentation of B-type dehydrocatechin dimer 
Another type of dimer formed during autoxidation is the A-type dehydrodicatechin. These compounds have $\mathrm{C}-\mathrm{O}-\mathrm{C}$ ether-type interflavan linkage. For retention time of 21.59 minutes the $m / z 577=M+1$ and $m / z 599=$ $\mathrm{M}+23\left(\mathrm{M}_{\mathrm{Na}+}\right)$ were assumed. Fragmentation mechanisms for obtaining the daughter fragments $\mathrm{m} / \mathrm{z} 425, \mathrm{~m} / \mathrm{z} 151, \mathrm{~m} / \mathrm{z} 245 \mathrm{from} \mathrm{m} / \mathrm{z} 577, \mathrm{~m} / \mathrm{z} 360$ were proposed. Scheme 3. shows the proposed fragmentation path for a A-type dehydrodicatechin. It was also explained the formation of $m / z 561$ as $\mathrm{M}-\mathrm{H}_{2} \mathrm{O}+\mathrm{H}^{+}$ and $m / z 463$ as $m / z 449+\mathrm{CH}_{3} \mathrm{OH}-\mathrm{H}_{2} \mathrm{O}$.Fragments with a mass greater than $\mathrm{m} / \mathrm{z}$ 599 may be adducts of the dimer with solvents and other molecules. Other fragments were obtained as follows: $m / z \quad 447=424+23\left(\mathrm{M}_{\mathrm{Na}+}\right), \mathrm{m} / z 217=$ $\mathrm{m} / \mathrm{z} 245$ - Mco.

In order to analyze the kinetics of catechin autoxidation by atmospheric oxygen, the accumulation of oxidation products at different $\mathrm{pH}$ values was recorded in time. During the autoxidation of catechin the effect of $\mathrm{pH}$ cannot be ruled out, as the basic $\mathrm{pH}$ should increase the proportion of phenolate anions leading to a larger quantity of reaction dimers than the protonated phenol. Figure 3 A shows absorption spectra for dimers at different pHs. The characteristic absorbance of catechin reaction products indicated a bathochromic shift with the $\mathrm{pH}$ increase. The maxima present at 280 and 430 are characteristic for the autoxidation products obtained at neutral and basic $\mathrm{pH}$ and at 280 and $390 \mathrm{~nm}$ for $\mathrm{pH} 5.5$ reaction products.

The characteristic bands of the dimers were used for further kinetic analysis by monitoring and recording consecutive spectra for 90 minutes.

The UV-Vis spectra of catechin solution mixed with acidic buffer presents a minimal variation where dehydrocatechin dimers have characteristic wavelengths of 280 and $390 \mathrm{~nm}$. For neutral and basic pHs the reaction takes place with a decrease in absorbance at 280 and an increase at $430 \mathrm{~nm}$.

The dimers concentrations were calculated from their absorbance. The kinetic curves of dimer concentration ([Dimer]) as function of time are shown in Figure $3 \mathrm{~B}$. Using the initial linear dependence of the kinetic curves [Dimers] $=f(t)]$, the initial reaction rates for the autoxidation of catechin, in methanolic solutions with buffers of various $\mathrm{pH}$ were estimated. The results are presented in Table 1. In mild alkaline media $(\mathrm{pH}=8)$ the conversion of catechin in dimers is maximum. 
ANCA - RUXANDRA LEONTIEŞ, ADINA RĂDUCAN, IOANA CATALINA GÎFU, DAN FLORIN ANGHEL
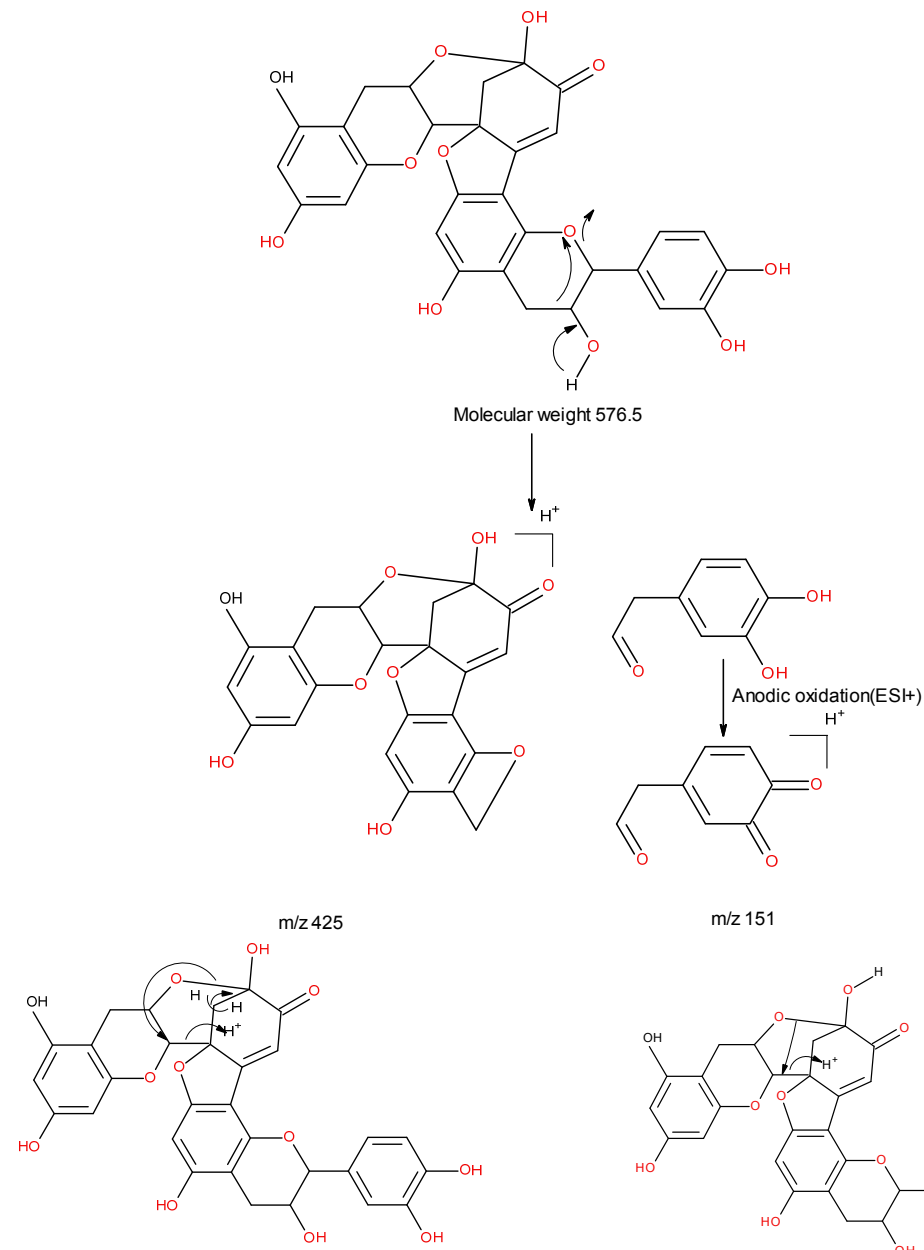

$\mathrm{m} / \mathrm{z} 151$
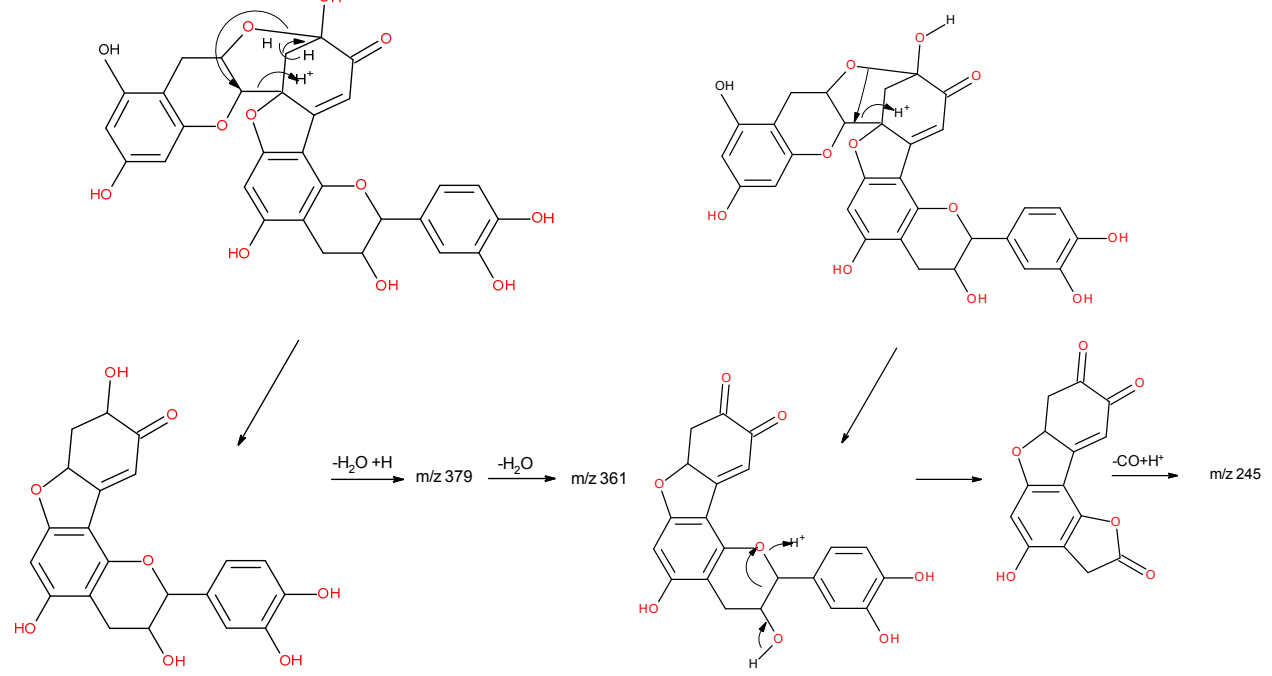

Scheme 3. Fragmentation of A type dehydrocatechin dimer 

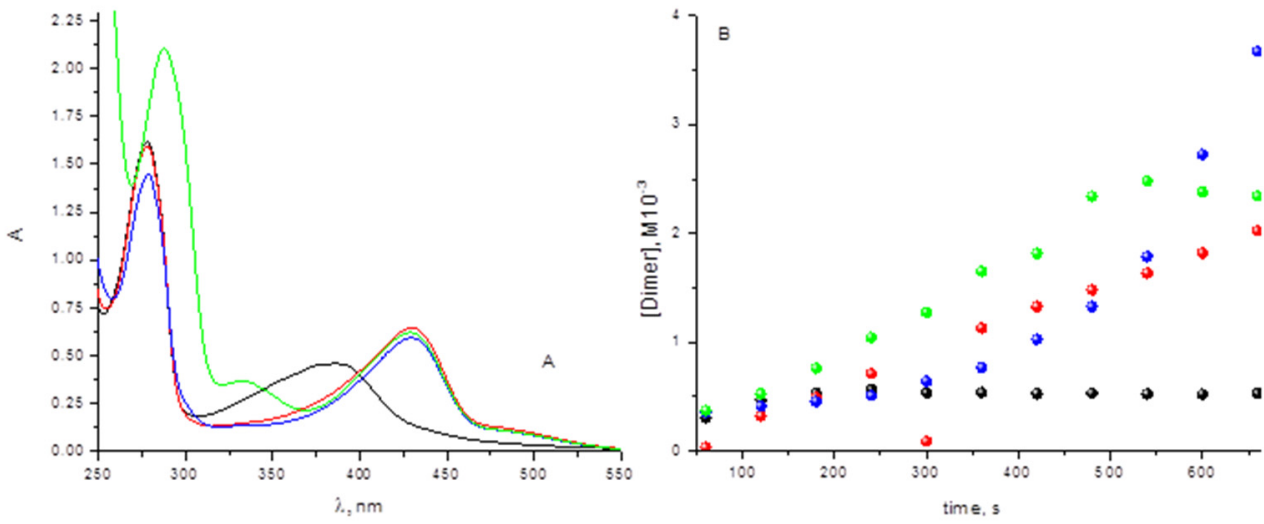

Figure 3. (A) Dimers spectra at different $\mathrm{pHs}$ and (B) Kinetic curve for dimer formation in catechin autoxidation process at different pHs: 5.5 (black), 7 (red), 8 (blue), 10 (green)

Table 1. The variation of the initial reaction rate with the $\mathrm{pH}$ for the autooxidation reaction of catechin solutio

\begin{tabular}{|c|c|}
\hline $\mathbf{p H}$ & $\left.\mathbf{V}^{\mathbf{0}} \cdot \mathbf{1 0} \mathbf{8}^{8} / \mathbf{M s}^{-1}\right)$ \\
\hline 5.5 & $(0.241 \pm 0.072)$ \\
\hline 7.2 & $(3.05 \pm 0.07)$ \\
\hline 8.0 & $(5.31 \pm 0.12)$ \\
\hline 9.9 & $(0.299 \pm 0.042)$ \\
\hline
\end{tabular}

\section{CONCLUSIONS}

The autoxidation of catechin was investigated in buffered methanolic solutions. The analysis of the reaction mixture indicates the presence of catechin dimers (dehydrocatechin $A$ and $B$ ) with similar structures and properties. From the analysis of the UV-VIS absorption spectra, the dimers characteristic bands were identified and further used for the kinetic analysis. It was found that in catechin autoxidation the dimer formation is favored at $\mathrm{pH}$ 8. The results are in agreement with other literature data confirming an oxidation mechanism implying dimers as products, with intermediate formation of semiquinons. 
ANCA - RUXANDRA LEONTIEŞ, ADINA RĂDUCAN, IOANA CATALINA GÎFU, DAN FLORIN ANGHEL

\section{EXPERIMENTAL SECTION}

\section{Materials}

Methanol and glacial acetic acid were HPLC grade. Deionized water ( $<18 \mathrm{M}$ resistance) was obtained from a Milli-Q Element water purification system (Millipore, Bedford, MA). Catechin and all the other chemicals were purchased from Sigma Aldrich.

\section{Preparation of autoxidation products of catechin}

In order to analyse the products resulting from the catechin autoxidation process, catechin was solubilized in methanol and a $0.1 \mathrm{mM}$ solution was obtained. The catechin solution was diluted with phosphate buffer $(0.1 \mathrm{M}, \mathrm{pH}$ 8) to a final concentration of $0.03 \mathrm{mM}$ in catechin. The final mixture $(100 \mathrm{~mL})$ was incubated in dark at room temperature, in the presence of atmospheric oxygen. After 24 hours, the mixture was washed with ethyl acetate. The upper layer was collected and dried using a rotary evaporator with a water bath at $40^{\circ} \mathrm{C}$ for 40 minutes, during which complete evaporation of the ethyl acetate was carried out. The $0.02 \mathrm{mg}$ of yellow powder obtained in the flask was solubilized in $2.5 \mathrm{~mL}$ of methanol.

\section{Liquid chromatography-mass spectrometry}

A Finnigan MAT Spectra System P4000 pump was used coupled with a UV6000LP diode array detector and a Finnigan AQA mass spectrometer. Analyses were carried out on a Superspher 100-RP18, $125 \cdot 2 \mathrm{~mm}, 4 \mu \mathrm{m}$, column (Macherey-Nagel, Germany), protected by a guard column packed with the same material, and maintained at $40^{\circ} \mathrm{C}$. Analyses were carried out employing electrospray ionization (ESI) at the positive ion mode, with acquisition set at 12 and $80 \mathrm{eV}$, capillary voltage $3.5 \mathrm{kV}$, source voltage 4.9 $\mathrm{kV}$, detector voltage $650 \mathrm{~V}$ and probe temperature $400 \mathrm{C}$. Eluent $(\mathrm{A})$ and eluent (B) were $2.5 \%$ aqueous acetic acid solution and methanol, respectively. The flow rate was $0.300 \mathrm{~mL} \cdot \mathrm{min}^{-1}$, and the elution program used was as follows: $0-15 \mathrm{~min}, 80 \%$ A. $20 \% \mathrm{~B} ; 15-25 \mathrm{~min}, 100 \% \mathrm{~B}$. The injection volume was $2 \mu \mathrm{L}$.

\section{UV-Vis spectrophotometry}

A stock solution of catechin in methanol $10^{-3} \mathrm{M}$ was prepared. The dilution was made with buffer at $\mathrm{pH} 5.5,7,8$ and 9.9 to a final concentration of $1.5 \cdot 10^{-4} \mathrm{M}$. Catechin autoxidation process was recorded using a JASCO V-350 spectrophotometer. Kinetic studies in the presence of isolated strawberry peroxidases [9] have shown that the product formed at $5.5 \mathrm{pH}$ has 
a molar absorption coefficient $\varepsilon=4600 \mathrm{M}^{-1} \mathrm{~cm}^{-1}$. This value was used to determine the concentration of dimers at this $\mathrm{pH}$ and then extrapolated to the rest of $\mathrm{pH}$, under conditions where Lambert-Beer law was tested for the range of concentrations considered. From the absorbance characteristic of the dimer wavelengths, the concentration was calculated. For catechin autoxidation process, the characteristic absorbance of the dimers at $430 \mathrm{~nm}$ was measured in time to obtain extended kinetic curves and estimate the initial reaction rates.

\section{REFERENCES}

1. J.M. Landete, Critical Review in Food Science and Nutrition, 2012, 52, 936.

2. M. Friedman, Journal of Agricultural and Food Chemistry, 2014, 62, 6025.

3. T. Tanaka, Y. Matsuo, I Kouno, Internationa Journal of Molecular Science, 2010, 11, 14.

4. S. Guyot, V.B. Chewier, J.M. Souquet, M.Moutounet, Journal of Agricultural and Food Chemistry, 1995, 43, 2458.

5. E.L. da Silva, D.S.P. Abdalla, J. Terao, IUBMB Life, 2000, 49, 289.

6. M.K. Chahar, N.Sharma, M.P. Dobhal, Y.C. Joshi, Pharmacognosy Reviews, 2011, 5, 1.

7. S.C. Forester, J.D. Lambert, Molecular Nutrition \& Food Research, 2011, 55, 844.

8. L. Pourcel, J.M. Routaboul, V.Cheynie, L.Lepiniec, I. Debeaujon, Trends in Plant Science, 2006, 12, 29.

9. Reaction, M. Lopez-Serrano, A. Ros Barcelo, Journal of Agricultural and Food Chemistry, 2002, 50, 1218. 
